\title{
Care-Koordinatorin: \\ Resultate einer Pilotstudie
}

\section{Maria M. Wertli; Thomas Beck; Kristina Tänzler; Manuela Steinacher; Martin Perrig; Drahomir Aujesky}

Departement für Allgemeine Innere Medizin, Inselspital, Universität Bern

\section{Hintergrund}

Eine Befragung im Rahmen einer Begleitstudie zur Einführung der SwissDRG zeigte, dass die administrative Belastung der akutsomatisch tätigen Ärztinnen und Ärzte (nachfolgend Ärzte) laufend zunimmt [1]. Besonders davon betroffen sind Assistenzärzte, die nur noch ca. einen Drittel ihrer Tagesarbeitszeit für patientennahe medizinische Tätigkeiten aufwenden [2]. Insbesondere an Universitätsspitälern hat das Ausmass an administrativen und koordinativen Funktionen der Assistenzärzte aufgrund der zunehmend anspruchsvollen Betreuung der multimorbiden und komplexen Patienten ein geradezu bedenkliches Ausmass erreicht. So zeigte eine prospektive Studie auf der Inneren Medizin am CHUV, dass die Assistenzärzte lediglich während 16\% ihrer Tagesarbeitszeit direkten Patien-

\section{Coordinatrice des soins: résultats d'une étude pilote}

La recherche concomitante à l'occasion de l'introduction de SwissDRG met en évidence une augmentation constante de la charge de travail administrative des médecins-assistants. Dans les cliniques de médecine interne générale qui accueillent essentiellement des patients polymorbides, au cas complexe, les tâches d'administration et de coordination des médecinsassistants ont pris des proportions considérables. Une étude prospective au service de médecine interne du CHUV a ainsi montré que ces derniers ne passent que $16 \%$ de leur temps de travail quotidien au contact direct des patients. A l'occasion d'un projet pilote prospectif contrôlé de 6 mois à la clinique universitaire de médecine interne de l'Inselspital, le recours à une coordinatrice des soins a été testé dans un service pilote, afin de délester les médecins-assistants sur le plan administratif. Les résultats ont montré que ceux-ci pouvaient déléguer environ $80 \%$ des travaux administratifs à la coordinatrice. Par ailleurs, une nette hausse de l'efficacité avec des quotients de durée de séjour significativement réduits et par conséquent des coûts par cas moindres a pu être démontrée dans le service pilote avec coordinatrice des soins par rapport aux services témoins qui en étaient dépourvus. Pour résumer, notre étude pilote a révélé que l'intervention d'une coordinatrice des soins dans une clinique de médecine interne peut permettre un délestage substantiel des médecins-assistants au niveau administratif, tout en améliorant les résultats. L'objectif de l'article ci-après est de détailler plus précisément le projet pilote. tenkontakt hatten [3]. Die Assistenzärzte verbrachten täglich 5,4 Stunden (46\% der Arbeitszeit) am Computer, um Patientenverläufe zu schreiben, Berichte zu verfassen oder für andere patientenbezogene administrative Tätigkeiten. Die verbleibende Tagesarbeitszeit verteilte sich auf andere administrative Aufgaben wie Versicherungsanfragen, Codierungsaufgaben sowie Lehre und Weiterbildung [1,3].

Bisherige Massnahmen zur administrativen Entlastung der Ärzte beinhalteten den Einsatz von Sekretariatspersonal, welches v.a. im Berichtswesen (Schreiben von Ein- und Austrittsberichten und z.T. Verlaufseinträgen, Bestellen von Vorberichten und -befunden) Entlastung schuf [4]. Die Anmeldung und Koordination von Untersuchungen und Konsilien sowie die inhaltliche Planung der Spitalentlassung und der Nachbetreuung (z.B. Kontaktaufnahme mit den nachbetreuenden Ärzten, Spitex, Rehabilitationen) sind in der Regel weiterhin Kernaufgaben der Assistenzärzte.

Im Ausland übernimmt zunehmend nichtärztliches Personal (Nurse Practitioners, Physician Assistants, Physician Associates) unter ärztlicher Aufsicht Aufgaben in der klinischen Patientenbetreuung, aber auch administrative Tätigkeiten wie Anfordern von Konsilien, radiologischen Untersuchungen und Labortests [5]. Gemäss informellen Berichten gibt es auch im Schweizer Spitalbereich mittlerweile verschiedene Modelle zur ärztlichen Entlastung durch geschultes nichtärztliches Personal. Zu den Beispielen gehören Clinical Nurses, Patienten-Coaches oder Nurse Practitioners auf internistischen und chirurgischen Kliniken, die unter ärztlicher Supervision Teile der koordinativ-administrativen und klinischen Stationsarbeit übernehmen, indem sie beispielsweise täglich Patienten visitieren, Angehörigengespräche führen, Medikamente verordnen und Konsilien und Untersuchungen anmelden. Obwohl informelle Rückmeldungen zu diesen Projekten positiv sind, fehlen unseres Wissens formelle Evaluationen zur Effektivität solcher Modelle. Um Nutzen und Kosten eines Einsatzes von nichtärztlichem Personal zur Entlastung der Assistenzärzte in der stationären Allgemeinen Inneren Medizin zu testen, führten wir auf einer Bettenabteilung der Universitäts- 


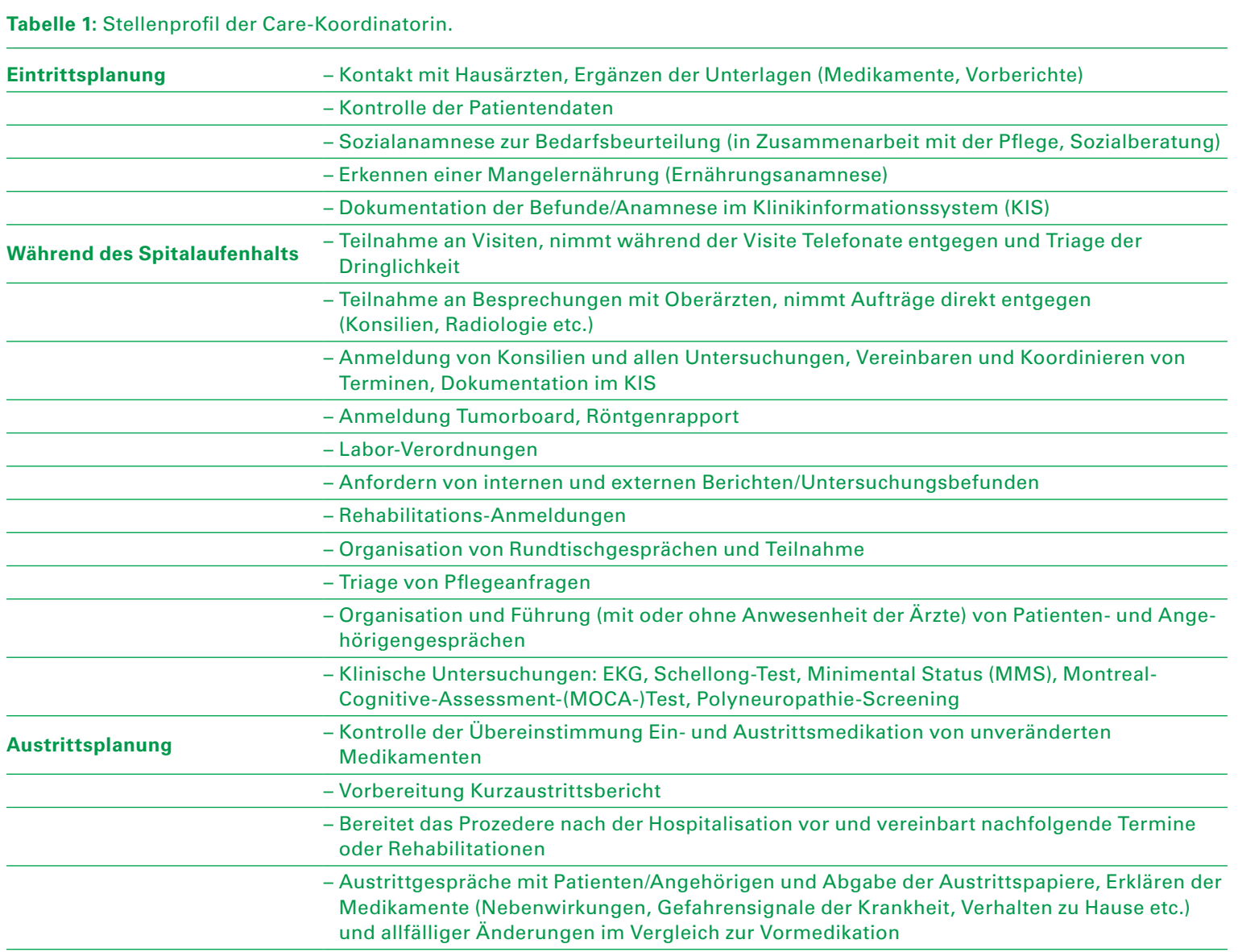

klinik für Allgemeine Innere Medizin des Inselspitals Bern ein 6-monatiges Pilotprojekt durch. Das Ziel des Pilotprojekts war es, den Impact einer Care-Koordinatorin auf die administrative Entlastung der Assistenzärzte, Behandlungseffizienz und Kosten zu untersuchen.

\section{Methoden}

Wir führten auf einer Abteilung mit 17 Betten der Universitätsklinik für Allgemeine Innere Medizin des Inselspitals Bern von Februar bis Juli 2016 ein prospektives, kontrolliertes Pilotprojekt zum Einsatz einer Care-Koordinatorin (100 Stellenprozente) durch. In einem ersten Schritt rekrutierten und schulten wir eine erfahrene medizinische Praxisassistentin (10 Jahre Berufserfahrung). Ihre administrativen und klinischen Tätigkeitsbereiche wurden in einem Stellenprofil definiert (Tab. 1), welches im Verlauf des Projekts schrittweise weiterentwickelt wurde. Untersucht wurden folgende Endpunkte: (1) die administrative Entlastung und Zufriedenheit der Assistenzärzte mit der CareKoordinatorin, (2) Effizienzparameter und (3) Kosten. Die geschätzte administrative Entlastung und die $\mathrm{Zu}$ friedenheit mit der Care-Koordinatorin während der Pilotphase wurde mit einem freiwilligen, anonymen
Fragebogen erfasst. Die Fragen umfassten Angaben zum geschätzten Ausmass der administrativen Entlastung, der Effizienz und Qualität der durch die CareKoordinatorin übernommenen Tätigkeiten sowie der Fehlerhäufigkeit.

Die Effizienzparameter und Kosten der Pilotabteilung während der Pilotperiode (02-07/2016) wurden zeitlich parallel mit Kontrollabteilungen (total 79 Betten) ohne Care-Koordinatorin verglichen. Sowohl die Pilot- wie auch die Kontrollabteilungen nahmen allgemeininternistische Patienten hauptsächlich vom Universitären Notfallzentrum auf. Die untersuchten Effizienzparameter waren der Verweildauer-Quotient (VDQ), ein Mass für die Verweildauer im Vergleich zur statistischen mittleren Verweildauer bei gleichem DRG in der Schweiz. Der VDQ ist weniger empfindlich auf Veränderungen des Casemix als die mittlere Verweildauer (VD) und korreliert mit der Rentabilität [6]. Ein VDQ von $<1$ spricht für eine überdurchschnittliche und ein Wert $>1$ für eine unterdurchschnittliche Effizienz. Weiter analysierten wir die mittlere Fallkomplexität mittels Patient Clinical Complexity Level (PCCL) unter SwissDRG. Der PCCL wird aufgrund der Nebendiagnosen ermittelt (Spannweite 0 bis 4 Punkte). Wir untersuchten die mittleren, schweregradkorrigierten Fall- 


\begin{tabular}{|c|c|}
\hline Fragen & Mittelwert (SD) ${ }^{1}$ \\
\hline Die Care-Koordinatorin entlastet/unterstützt meine Alltagsarbeit & $9,4(1,1)$ \\
\hline $\begin{array}{l}\text { Die verlangten Administrativarbeiten konnten alle abgegeben } \\
\text { werden }\end{array}$ & $7,9(2,0)$ \\
\hline Die Arbeiten wurden im verlangten Zeitrahmen erledigt & $9,6(0,5)$ \\
\hline Die Arbeiten wurden in der gewünschten Qualität erledigt & $8,9(0,9)$ \\
\hline $\begin{array}{l}\text { Fehler/Probleme wegen unzureichender Kommunikation zwischen } \\
\text { Assistenzarzt und Care-Koordinatorin }\end{array}$ & $3,0(2,0)$ \\
\hline
\end{tabular}

kosten pro Case-Mix-Punkt (CMP) und das mittlere finanzielle Ergebnis unter SwissDRG pro Fall unter der Annahme einer Basisrate von 11000 CHF. Sämtliche Effizienzparameter wurden dem zentralen MedizinControlling des Inselspitals entnommen. Zudem wurde der mögliche Einfluss der Care-Koordinatorin auf selbstdeklarierte Überstunden analysiert, wobei die durchschnittliche Überzeit pro Fall auf der Pilotstation 02-07/2016 mit derjenigen von 10/2015-01/2016 verglichen wurde. Die Vergleichsanalysen wurden mittels Wilcoxon-Rangsummentest durchgeführt, wobei ein p $<0,05$ als statistisch signifikant angesehen wurde. Wir verglichen die gemittelten VDQ pro Fall und Fallkosten pro CMP, da dadurch Ausreisser einen geringeren Einfluss haben [7]. Die statistischen Analysen erfolgten mit Stata für Windows, Version 14.2.

\section{Resultate}

Insgesamt beantworteten 7 der 8 (87\%) auf der Pilotabteilung eingesetzten Assistenzärzte den anonymisierten Fragebogen. Die Unterstützung im Alltag durch die Care-Koordinatorin wurde als sehr gross eingeschätzt, im Durchschnitt konnten 79\% der anfallenden administrativen Arbeiten an die Care-Koordinatorin delegiert werden. Gemessen auf einer Skala von 1 bis 10 wurde die Arbeit der Care-Koordinatorin als sehr gut ( $\geq 7,8$ Punkte) bei niedrigem Fehlerpotential (3,0 Punkte) evaluiert (Tab. 2). Informelle Rückmeldungen seitens von Pflege und Sozialberatung waren ebenfalls äusserst positiv.

Während der Pilotperiode (02-07/2016) wurden auf der Pilotabteilung 361 Patienten stationär behandelt, auf den Kontrollabteilungen 1426 Patienten. Der Case Mix Index (CMI) auf der Pilotabteilung und den Kontrollabteilungen war vergleichbar (1,09 vs. 1,2; $p=0,3)$. Der Vergleich der Pilotabteilung gegenüber den Kontrollabteilungen ohne Care-Koordinatorin während der Pilotperiode zeigte bei vergleichbarem PCCL (Fallkomplexität) eine statistisch signifikante Abnahme des durchschnittlichen VDQ $(-0,12)$ sowie den Trend einer Ab- nahme der durchschnittlichen VD (-0,8 Tage) (Tab. 3). Weiterhin liess sich ein Trend zur Abnahme der durchschnittlichen Fallkosten pro CMP (559 CHF) sowie eine statistisch nicht-signifikante Zunahme des Gesamtergebnisses pro Fall (480 CHF) zugunsten der Pilotabteilung feststellen. Gegenüber der Vorperiode (10/201501/2016) ohne Care-Koordinatorin gingen auf der Pilotabteilung während der Pilotphase (02-07/2016) die Überzeiten von 1,25 auf 1,08 Std. (17 Min.) pro Fall zurück.

\section{Diskussion}

In unserer Pilotstudie führte der Einsatz einer CareKoordinatorin zu einer substantiellen administrativen Entlastung der Ärzte, die Arbeit der Care-Koordinatorin wurde als sehr positiv beurteilt. Gegenüber Abteilungen ohne Care-Koordinatorin zeigte sich bei vergleichbarer Fallschwere und -komplexität eine signifikante Verbesserung des VDQ und ein Trend zur Abnahme der VD als Hinweis für eine Effizienzsteigerung. In Analogie zu unseren Resultaten empfanden in einer Umfrage aus dem Vereinigten Königreich mehr als 80\% der befragten Ärzte die Zusammenarbeit mit Physician Assistants als positiv [8]. Speziell hervorgehoben wurden dabei die verbesserte Kontinuität der Patientenbetreuung, eine grössere Flexibilität und Entlastung des Behandlungsteams sowie eine grössere Patientenzufriedenheit.

Die zunehmende administrative Belastung in der Behandlung stationärer multimorbider Patienten bedarf neuer Ansätze zur Unterstützung des Behandlungsteams. Es gibt Hinweise, dass der zunehmende administrative Aufwand nicht nur die Zufriedenheit der Ärzte, sondern auch die Qualität der Weiterbildung und Patientenbehandlung negativ beeinflusst $[9,10]$. In

Die zunehmende administrative Belastung in der Behandlung stationärer multimorbider Patienten bedarf neuer Ansätze zur Unterstützung des Behandlungsteams.

einer Befragung von Schweizer Assistenz- und Oberärzten waren $80 \%$ der Meinung, dass viele administrative Aufgaben gut delegierbar wären und damit 4-6 Stunden Arbeit pro Woche eingespart werden könnten [11]. Das von uns vorgestellte Modell einer Care-Koordinatorin, die eng mit den Abteilungsärzten zusammenarbeitet, führte nebst einer spürbaren administrativen Entlastung der Ärzte auch zu einer signifikanten Abnahme des VDQ und zu weniger Überzeiten. Dies dürfte primär auf die bessere Koordination der Abklärungen und Austrittsplanung zurückzuführen sein. Da die Spitalaufenthaltsdauer einen massgeblichen Kostenfaktor darstellt, ist der Einsatz einer Care- 


\begin{tabular}{|c|c|c|c|}
\hline & Pilotabteilung & Kontrollabteilungen & P-Wert $^{1}$ \\
\hline & \multicolumn{2}{|c|}{ Mittelwert (Standarddeviation) } & \\
\hline Mittlerer Verweildauer-Quotient ${ }^{2}$ & $0,95(0,7)$ & $1,07(0,9)$ & 0,02 \\
\hline Mittlere Verweildauer (Tage) & $7,1(6,0)$ & $7,9(7,4)$ & 0,06 \\
\hline Mittlerer Patient Clinical Complexity Level (Punkte) & $2,56(1,5)$ & $2,54(1,5)$ & 0,76 \\
\hline Mittlere Fallkosten/Case-Mix-Punkt (CHF) & 11843 & 12402 & 0,10 \\
\hline Mittleres Ergebnis/Fall (CHF) ${ }^{5}$ & -1148 & -1628 & $\overline{0,16}$ \\
\hline
\end{tabular}

1 Vergleich zwischen Pilotabteilung mit Care-Koordinatorin und Kontrollabteilungen ohne Care-Koordinatorin während der Pilotperiode (02-07/2016).

2 Gemittelte Summe der Verweildauer-Quotienten pro Fall (<1, überdurchschnittliche Effizienz; >1, unterdurchschnittliche Effizienz).

3 Fallkomplexität unter SwissDRG, ermittelt durch Nebendiagnosen (0 Punkte, tiefe Komplexität; 4 Punkte, hohe Komplexität).

4 Gemittelte Summe der Kosten pro Case-Mix-Punkt pro Fall. Liegt der Wert über der hier angenommenen Basisrate von 11000 CHF, sind die Fälle defizitär.

5 Mittleres Ergebnis pro Fall unter SwissDRG, entspricht dem Gesamtgewinn oder -verlust der Abteilung pro Fall im beobachteten Zeitraum.

Koordinatorin auch ökonomisch interessant. Bei 361 auf der Pilotabteilung während der 6-monatigen Pilotperiode behandelten Patienten und einer mittleren, schweregradkorrigierten Kostenreduktion von 559 CHF pro Fall liesse sich extrapoliert auf ein Kalenderjahr eine Gesamtkostenreduktion von 403598 CHF auf der Pilotabteilung erreichen. Bei Jahreslohnkosten von 84978 CHF wäre der Einsatz der Care-Koordinatorin als kosteneffizient zu bezeichnen. Ob der Einsatz einer Care-Koordinatorin nach unserem Modell einen Einfluss auf die Behandlungsqualität hat, lässt sich in unserer Studie nicht beantworten und muss weiter untersucht werden.

\section{Einschränkungen}

Obwohl die in unserer Pilotstudie gezeigte Effizienzsteigerung ermutigend ist, müssen diese Resultate mit Vorsicht interpretiert werden. Da Effizienz auch durch individuelle Eigenschaften (Erfahrung, Arbeitsorganisation) des Ärzteteams beeinflusst wird, können wir nicht ausschliessen, dass Unterschiede zwischen Pilotund Kontrollabteilungen durch Unterschiede im Ärztemix (mit)verursacht wurden. Zudem dürfte die Effizienzsteigerung auch von individuellen Eigenschaften der eingesetzten Care-Koordinatorin und spezifischen Faktoren der Klinikorganisation abhängen, was die Reproduzierbarkeit unserer Resultate einschränkt. Bei vergleichbarem CMI und PCCL auf Pilot- und Kontrollabteilungen ist es hingegen unwahrscheinlicher, dass unsere Resultate durch einen unterschiedlichen Patientenmix erklärbar sind.

PD Dr. med. Maria M. Wertli, $\mathrm{PhD}$

Universitätsklinik für Allgemeine Innere Medizin Inselspital

Freiburgstrasse 8

CH-3010 Bern

Maria.Wertli[at]insel.ch nistischen Universitätsklinik zu einer substantiellen Administrativentlastung der Assistenzärzte, zu einer Effizienzsteigerung sowie wahrscheinlich zu einer Kostenreduktion führt. Basierend auf diesen Ergebnissen, plant die Universitätsklinik für Allgemeine Innere Medizin des Inselspitals, Care-Koordinatorinnen auf allen ihren Bettenstationen einzuführen und den Impact auf Arbeitszufriedenheit und Kosten-Effizienz systematisch zu untersuchen.

\section{Referenzen}

1 Golder L, et al. Trotz steigendem Dossieraufwand bleibt die Spitalärzteschaft motiviert. In: Begleitstudie anlässlich der Einführung von SwissDRG sowie der geplanten stationären Tarife in der Rehabilitation und Psychiatrie im Auftrag der FMH, 5. Befragung 2015, G. Bern, Editor. 2016, GFS Bern: Bern. p. 1-77.

2 Meyer B, et al. Administrativer Aufwand für Ärzte steigt weiter an. Schweiz Ärztezeitung. 2016;97(1):6-8.

3 Wenger N, et al. FM276: Quantitative and objective assessment of resident's workday organization: the Medical Day study [abstract]. Swiss Primary and Hospital Care, 2016. 16 Suppl(9): p. 21-2.

4 Schade V. Zum Kern ärztlichen Tuns. Schweiz Ärztezeitung, 2016;97(34):1138-41.

5 Abraham J, et al. Development and implementation of non-medical practitioners in acute care. Br J Nurs. 2016;25(20):1129-34.

6 Einkaufsgemeinschaft HSK Benchmark HSK 2016 für SwissDRG. 2016. 1-18.

7 Larivière V and Y Gingras. Averages of ratios vs. ratios of averages: An empirical analysis of four levels of aggregation. Journal of Informetrics. 2011;5(3):392-9.

8 Williams LE and TS Ritsema. Satisfaction of doctors with the role of physician associates. Clin Med (Lond). 2014;14(2):113-6.

9 Woolhandler S and DU Himmelstein. Administrative work consumes one-sixth of U.S. physicians' working hours and lowers their career satisfaction. Int J Health Serv. 2014;44(4):635-42.

10 Rao SK, et al. The Impact of Administrative Burden on Academic Physicians: Results of a Hospital-Wide Physician Survey. Academic Medicine, 2016. Publish Ahead of Print.

11 Verband Schweizer Assistenz- und Oberärztinnen und -ärzte (VSAO). Reduktion bzw. Delegation administrativer Aufgaben von Assistenz- und Oberärztinnen. 2016 September 2016 [cited 2016 December 7, 2016]; Available from: http://www2.vsao.ch/content/ default.asp?txtParentID $=452 \& \operatorname{txtCatID}=624$ 